\title{
Papers
}

\section{Risk factors for early onset neonatal group B streptococcal sepsis: case-control study}

\author{
Sam Oddie, Nicholas D Embleton, on behalf of the Northern Neonatal Network
}

\begin{abstract}
Objectives To quantify risk factors for and the prevalence of early onset group B streptococcal sepsis in neonates in a geographically defined population. Design Cases were collected prospectively for two years from April 1998 and compared with four controls each, matched for time and place of delivery. Setting The former Northern health region of the United Kingdom.

Participants Infants infected with group B streptococcus in the first week of life.

Results The prevalence of early onset group B streptococcal sepsis was 0.57 per 1000 live births. Premature infants comprised $38 \%$ of all cases and $83 \%$ of the deaths. Prematurity (odds ratio 10.4, 95\% confidence interval 3.9 to 27.6 ), rupture of the membranes more than 18 hours before delivery (25.8, 10.2 to 64.8 ), rupture of the membranes before the onset of labour (11.1, 4.8 to 25.9), and intrapartum fever (10.0, 2.4 to 40.8) were significant risk factors for infection. Had the interim recommendations on best practice issued by the Group B Streptococcus Working Group of the Public Health Laboratory Service been uniformly applied to the fetuses alive at the onset of labour, 29 of $37(78 \%)$ might have been given antibiotic prophylaxis during labour. At least 23 of these $29(79 \%)$ could have had antibiotics for four hours or more before delivery. To achieve this, $16 \%$ of all women would have been given antibiotics during labour.

Conclusions Early onset group B streptococcal sepsis remains an important problem in the United Kingdom. Prevention based on risk factors might reduce the prevalence at the cost of treating many women with risk factors. Using rupture of the membranes before the onset of labour as a risk factor might be expected to improve the success of guidelines for prophylaxis.
\end{abstract}

\section{Introduction}

Group B streptococcal disease is the leading cause of early onset neonatal sepsis in developed countries. ${ }^{1}$ Despite the widespread adoption of preventive strategies in the United States and Australia in recent years, ${ }^{2-4}$ uncertainty prevails as to whether early onset group B streptococcal sepsis is sufficiently common in the United Kingdom to justify widespread prophy- laxis. ${ }^{5}$ More recently the Group B Streptococcus Working Group of the Public Health Laboratory Service issued interim recommendations for best practice, to be used while further data are collected. ${ }^{67}$ Evidence from both the United States and Australia shows that the adoption of prophylactic policies significantly decreases the incidence. ${ }^{4}$.

Few data on the prevalence of early onset group B streptococcal sepsis in the United Kingdom, and no population based case-control studies on risk factors, are available. Such data as there are imply a prevalence of 0.5-1.15 cases per 1000 live births. ${ }^{9-14}$ Part of the variation in prevalence probably relates to differing characteristics of populations, although some variation is almost certainly related to case ascertainment and, possibly, differences in case definition. ${ }^{9}$ In addition, many neonates may become infected with group B streptococcus yet samples taken from them do not grow bacteria on culture. ${ }^{13}{ }^{15}$ Interpretation of epidemiological studies may be complicated by the use of antibiotic prophylaxis intra partum if local practices are not evaluated concurrently.

This study aimed to evaluate risk factors for early onset group B streptococcal sepsis by using a case-control design in a geographically defined population while considering preventive strategies already employed.

\section{Methods}

We contacted consultant paediatricians, senior neonatal nurses, consultant microbiologists, and scientific officers in microbiology at each of the 15 neonatal units throughout the former Northern region directly and asked them to cooperate with the study. We asked them to identify neonates of 24 weeks' gestation or more in whom group B streptococcus was isolated from a normally sterile site (blood or cerebrospinal fluid) in the first week of life. Cases were reported on a standardised form. The study included all neonates born between 1 April 1998 and 31 March 2000. We sent reminders of the study to individuals every six months. At the end of each 12 month period, we cross checked against cases notified to the Public Health Laboratory Service. We found no extra cases. We cross checked neonates who died from early onset group B streptococcal sepsis were against returns held by the local perinatal mortality survey, and any further cases that we found supplemented those already reported. ${ }^{16}$
Newcastle Neonatal
Service,
Department of
Child Health, Royal
Victoria Infirmary,
Newcastle upon
Tyne NE1 4LP
Sam Oddie
specialist registrar
Nicholas D
Embleton
specialist registrar
Correspondence to:
S Oddie
s.j.oddie@ncl.ac.uk
bmj.com 2002;325:308 
At the end of the study, we fed all notifications back to individuals and departments to ensure that all known cases had been reported to us. Microbiology departments with computerised databases were asked to provide a list of reported cases.

We selected four controls for each case in which the fetus was alive at the onset of labour. Controls were identified from records from the delivery suite from the same hospital as the case and comprised the two infants born alive immediately before and after each case. Intrapartum stillbirths were included in the case-control study but excluded from the prevalence calculations.

We reviewed obstetric, neonatal, and pathology case notes, paying particular attention to the reason for the onset of labour and the use of antibiotic prophylaxis during labour. Women were considered to have rupture of the membranes before the onset of labour if at least six hours elapsed between rupture of the membranes and the onset of regular uterine contractions.

A standardised questionnaire was sent to the clinical directors of obstetrics and senior clinical midwives at each of the region's 15 maternity units to identify written guidelines on the indications for and the use of antibiotic prophylaxis during labour, and to provide information on how often these guidelines were implemented in practice.

We made statistical comparisons by calculating odds ratios, sensitivities, and specificities using Epicalc 2000 version 1.12 and carried out logistic regressions using Minitab version 13. The study was carried out after consultation with the regional ethics committee for medical research.

\section{Results}

Thirty six liveborn babies developed group B streptococcal sepsis in the first week of life. As 62786 live births were registered in the study period, the prevalence is 0.57 per 1000 live births. In addition, three stillborn babies died of proved group B streptococcal infection, one during labour.

The cases presented early: 19 had symptoms by 1 hour of age and 26 by 24 hours of age. Just three babies developed symptoms after 48 hours of age. Twelve infants were admitted to neonatal units after resuscitation at birth, five had no symptoms but had blood cultures performed because of an identified risk factor, 13 developed respiratory symptoms, and four presented with seizures.

Thirty five infants had positive results on blood culture and two had positive cultures of cerebrospinal fluid. In one liveborn preterm infant infection was diagnosed at postmortem examination. Lumbar puncture was performed in 12 of 30 survivors, and meningitis was proved in three cases. Ten infants were ventilated, of whom six were less than 34 weeks' gestation. Five infants received inotropic support, and all died.

Six infants died in total. Five infants born at less than 36 weeks and presenting with symptoms of infection in the first hour died in the first 24 hours of life. One infant born at term presented with seizures on the third day and died on the 10th day.

In 17 liveborn cases (47\%) a change to instrumental delivery was undertaken during labour, in contrast with $25(17 \%)$ of the controls $(\mathrm{P}=0.001)$, which implies greater professional concern about the case fetuses than about the controls. Cases were more likely than controls to receive inflation by mask or intensive resuscitation at birth (20 cases, 13 controls, $\mathrm{P} \leqslant 0.001)$. Antenatal risk factors and unadjusted odds ratios are summarised in table 1.

We analysed the effect of the various risk factors on the prevalence of infection by using logistic regression (table 2). Two further models with four variables were developed to reflect the clinical situation more accurately. Each included only one variable relating to rupture of the membranes. In these analyses both pro-

Table 1 Risk factors for early onset group B streptococcal sepsis in cases and controls alive at the onset of labour

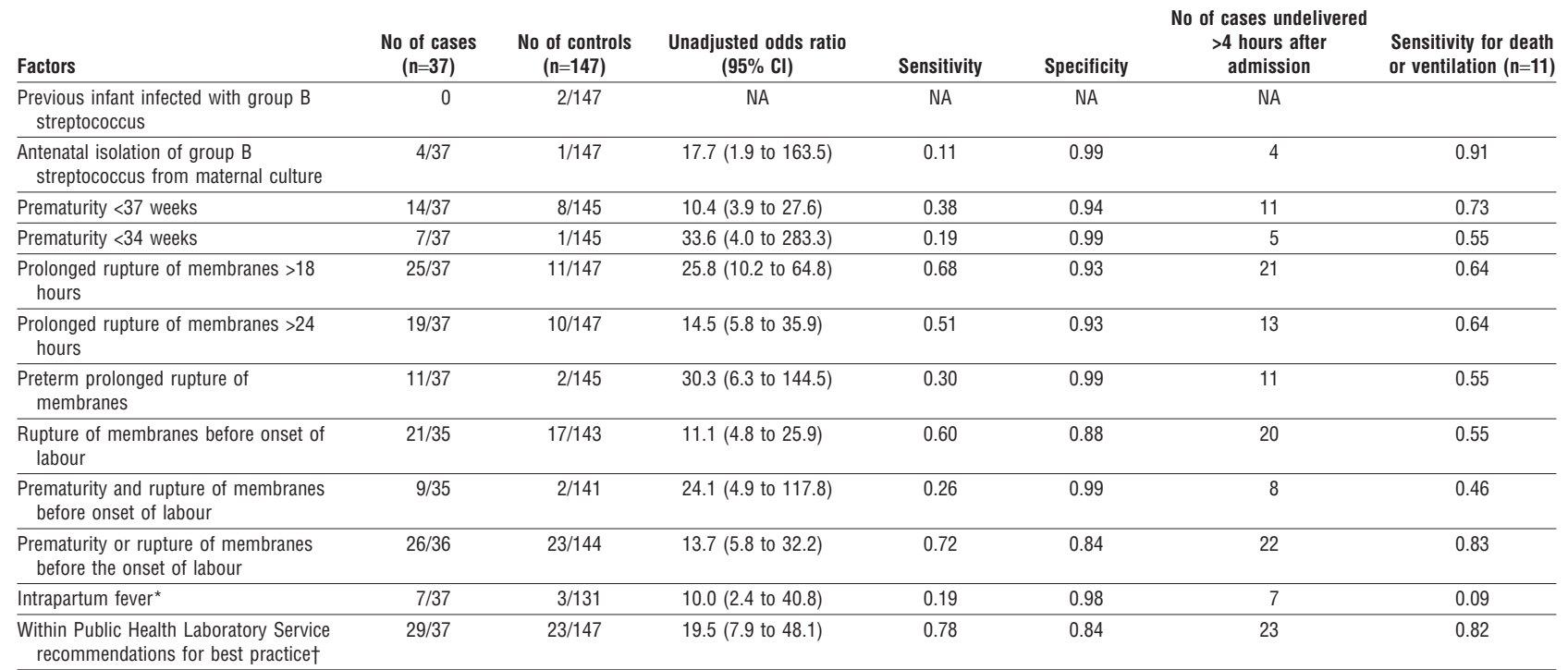

$\mathrm{NA}=$ not applicable.

${ }^{*}$ Defined as maternal fever of $38^{\circ} \mathrm{C}$ or above. Timing of fever not recorded.

†Antibiotic treatment intra partum should be considered for women who have previous infants with group B streptococcal infection, group B streptococcus isolated from the vagina or urine during pregnancy, chorioamnionitis, preterm prolonged rupture of the membranes, preterm labour, prolonged rupture of the membranes, or maternal fever. 
Table 2 Risk factors for early onset group B streptococcal sepsis: logistic regression model with five variables

\begin{tabular}{lc} 
Risk factor & $\begin{array}{c}\text { Adjusted odds ratio } \\
\text { (95\% CI) }\end{array}$ \\
\hline Group B streptococcus isolated during pregnancy & $1.9(0.03$ to 142.7$)$ \\
\hline Gestation $<37$ weeks & $12.1(2.7$ to 53.8$)$ \\
\hline Prolonged rupture of membranes $>18$ hours & $4.8(0.98$ to 23.1$)$ \\
\hline Rupture of membranes before onset of labour & $3.6(0.7$ to 17.6$)$ \\
\hline Intrapartum fever & $10.0(1.7$ to 60.7$)$ \\
\hline
\end{tabular}

longed rupture of the membranes (odds ratio 13.7, $95 \%$ confidence interval 4.8 to 39.5 ) and prelabour rupture of the membranes (10.7,3.8 to 30.1) are shown to be independent, statistically significant risk factors for infection. Prelabour rupture of the membranes was a risk factor that was satisfied at a median 26.7 hours before delivery of the infant, compared with prolonged rupture of the membranes, which was satisfied at a median 13.5 hours before delivery.

We applied the criteria from the Public Health Laboratory Service's recommendations for best practice sequentially to the cases and controls (table 3 ). ${ }^{6}$ Of the 29 cases to which the criteria would have applied, review of medical notes showed that 23 were in hospital long enough before delivery to have received antibiotic prophylaxis for at least four hours before delivery. Application of these criteria might have resulted in treatment of $23(16 \%, 10 \%$ to $22 \%)$ control mothers. Intrapartum temperature was not clearly documented in 16 controls.

Complete data on the use of antibiotic prophylaxis during labour were available from 13 units. Eleven units had a written policy. Only two units said that it would be very likely or universal that women with prolonged rupture of the membranes beyond 18 hours would be treated with antibiotics.

Among the cases in this series only four infants had been exposed to antibiotics; chorioamnionitis was documented in the mother's notes for only two of these cases. In contrast just four of 147 control women had been given antibiotics during labour. Only two of these controls seem to have met the criteria of the Public Health Laboratory Service.

\section{Discussion}

This study shows that $78 \%$ of cases of early onset group B streptococcal sepsis occurring in the Northern region had the opportunity to receive prophylaxis during labour had the guidelines for best practice been applied rigorously. Such rigorous application of the guidelines would, however, have resulted in antibiotics being given to $16 \%$ of all women in labour.

\section{Potential limitations}

This population based study is likely to have recorded most liveborn infants with group B streptococcal infection because of its multiple ascertainment techniques. But as only about $65 \%$ of stillbirths underwent autopsy, some stillborn cases may not have been identified. ${ }^{17}$ Antenatal screening for group B streptococcus was not widespread during the study period, nor were enriched culture media in common use. Had cases been included in which supportive evidence of group B streptococcal sepsis was strong but deep cultures were not positive the recorded rate of infection would have been much higher. Considering the cases proved on culture in isolation will therefore result in an understimate of the true burden of disease. ${ }^{13}$ Our measured rate of infection is similar to that of other studies in the United Kingdom, and the observed variation may be explained by the case ascertainment and population characteristics. ${ }^{9}$ Other studies based in the United Kingdom have not analysed the presence of risk factors in a similar way.

\section{Risk factors}

Delivery at less than 34 weeks was a significant risk factor among cases (odds ratio 33.6), and in addition five of the six infants who died were preterm. Although this study is too small to define mortality accurately, at 0.94 deaths per 10000 live births the rate is similar to that previously quoted for the United States, Europe, and Australasia. ${ }^{18}$

The frequency of change to instrumental delivery and resuscitation at birth illustrates that many of the cases were probably infected in utero. For this reason antibiotic prophylaxis during labour should be started as soon as possible after risk factors have been identified. ${ }^{2}$ Published data show that giving intrapartum antibiotic prophylaxis for four hours significantly reduces the chances of early onset group B streptococcal sepsis in neonates after high risk labour. ${ }^{19} 20$ This study implies that most $(78 \%)$ case women giving birth who were at high risk according to the criteria of the Public Health Laboratory Service could have been identified in time to give at least four hours of antibiotic prophylaxis before delivery.

Table 3 Sequential retrospective application of the Public Health Laboratory Service's interim recommendations for best practice to cases and controls

\begin{tabular}{|c|c|c|c|c|}
\hline \multirow[b]{2}{*}{ Criteria } & \multicolumn{2}{|r|}{ Cases } & \multicolumn{2}{|r|}{ Controls } \\
\hline & $\begin{array}{c}\text { No } \\
(n=37)\end{array}$ & $\begin{array}{c}\text { Cumulative No (\%) potentially offered } \\
\text { prophylaxis during delivery }\end{array}$ & $\begin{array}{c}\text { No } \\
(n=147)\end{array}$ & $\begin{array}{c}\text { Cumulative No (\%) potentially offered } \\
\text { prophylaxis during delivery }\end{array}$ \\
\hline Previous infant infected with group B streptococcus & 0 & $0(0)$ & 2 & $2(1.4)$ \\
\hline $\begin{array}{l}\text { Group B streptococcus isolated in vagina in } \\
\text { pregnancy }\end{array}$ & 4 & $4(11)$ & 1 & $3(2.0)$ \\
\hline Group B streptococcal bacteruria in pregnancy & 0 & $4(11)$ & 0 & $3(2.0)$ \\
\hline Chorioamnionitis & 1 & $5(14)$ & 2 & $5(3.4)$ \\
\hline Preterm prolonged rupture of membranes & 11 & $14(38)$ & 0 & $5(3.4)$ \\
\hline Premature labour & 14 & $17(46)$ & 8 & $11(7.5)$ \\
\hline Prolonged rupture of the membranes & 25 & $28(76)$ & 11 & $21(14.3)$ \\
\hline Intrapartum fever & 7 & $29(78)$ & 3 & $23(15.6)$ \\
\hline
\end{tabular}




\section{Conclusion}

This study confirms the importance of well established antenatal risk factors for early onset group B streptococcal sepsis and shows that rupture of the membranes before the onset of labour is an important risk factor. Although prelabour rupture is strongly associated with prolonged rupture of the membranes and is not an independent risk factor, its use in guidelines for prophylaxis may make it possible for antibiotics to be prescribed at an earlier stage. Its occurrence is usually known well before delivery, unlike prolonged rupture of the membranes, which can often be determined with certainty only after delivery. ${ }^{21}$ Combinations of these risk factors will "predict" risk in more cases.

Any risk factor based strategy of antibiotic prophylaxis during labour should concentrate on risk factors most prevalent in the infected population while minimising the number of women overall who are exposed to antibiotics. The Public Health Laboratory Service's recommendations for best practice can be applied in different ways depending on the definitions used for prolonged rupture of the membranes and intrapartum fever. ${ }^{6}$ Had the criteria been applied as aggressively as here (for example, counting 19 hours of rupture of the membranes as a risk factor), intrapartum antibiotic prophylaxis would have been offered to $16 \%$ of women in labour and might have prevented or ameliorated infection in almost three quarters of the cases.

Realistically, however, the criteria might well be variably or incompletely applied, not all eligible women might accept antibiotics during labour, and prophylaxis might be ineffective. ${ }^{22}$ This interpretation of our data might therefore overestimate the potential effect of implementing the current recommendations rigorously. In addition, universal use of antibiotics is not without problems. Prophylactic strategies do not seem to have increased the prevalence of resistant organisms on the neonatal intensive care unit, ${ }^{4}$ but occasional reports have found that resistant coliforms may now be more common because of such an approach. ${ }^{23}$ Recent data also show that the use of broad spectrum intrapartum antibiotics might increase the incidence of necrotising enterocolitis. ${ }^{24}$ Guidelines should therefore seek to minimise the overall number of women exposed. This study provides data that will aid the development of such guidelines in the United Kingdom.

We thank everyone who contributed to this survey and in particular members of the Northern Neonatal Network, the medical records staff and Alice Downes, Jim Dodd, Roshan Adappa, Diane Snowden, and Dominic Sailor for retrieving the notes; and Edmund Hey, Louise Parker, and Unni Wariyar for support with the study design and the writing of this paper.

Contributors: NDE had the idea for the study, collected the cases, and carried out the survey. SO and NDE together planned the case-control aspects of the study of the use of intrapartum antibiotic prophylaxis. SO reviewed the clinical records, collated and analysed the data and wrote the first draft of the paper, which was completed by both authors. SO is the guarantor for the paper.

Funding: Northern Neonatal Network

Competing interests: None declared.

1 Baker C, Edwards M. Group B streptococcal infections. In: Remington J, Klein J, eds. Infectious diseases of the fetus and newborn infant. 5th ed. Philadelphia: Saunders, 2001:1091-156.
What is already known on this topic

Group B streptococcal infection is the leading cause of neonatal sepsis in the United Kingdom and an important, yet potentially preventable, cause of death

The prevalence of early onset group B streptococcal sepsis in the United Kingdom is not well defined

Data from the United States and Australia show that the prevalence may be reduced drastically by using selective antibiotic prophylaxis during labour

\section{What this study adds}

Odds ratios for established risk factors, calculated for a British population, might aid the development of prophylactic guidelines

Rupture of the membranes before the onset of labour should be considered as an important risk factor and might identify potential cases at an earlier stage

Current prophylactic guidelines might prevent or ameliorate three quarters of all cases of infection at the cost of giving antibiotics to $16 \%$ of all women in labour

2 American Academy of Pediatrics Committee on Infectious Diseases and Committee on Fetus and Newborn. Revised guidelines for prevention of early-onset group B streptococcal (GBS) infection. Pediatrics 1997:99:489-96.

3 Committee on Obstetric Practice. American College of Obstetrics and Gynaecologists ACOG committee opinion. Prevention of early-onset group B streptococcal disease in newborns. Int J Gynaecol Obstet 1996;54:197-205.

4 Jeffery HE, Lahra MM. Eight-year outcome of universal screening and intrapartum antibiotics for maternal group B streptococcal carriers. Pediatrics 1998;101:E2.

5 Isaacs D. Prevention of early onset group B streptococcal infection: screen, treat, or observe? Arch Dis Child Fetal Neonatal Ed 1998;79:F81 F82.

6 PHLS Group B Streptococcus Working Group. Interim "best practice" recommendations for the prevention of neonatal group B streptococcal infection in the UK. London: Central Public Health Laboratory, 2000. www.bapm-london.org/publications/gbs.pdf (accessed 13 May 2002).

7 McCartney C. Group B streptococci for neonatal disease. Lancet 2001;357:395.

8 Schrag SJ, Zywicki S, Farley MM, Reingold AL, Harrison LH, Lefkowitz $\mathrm{LB}$, et al. Group B streptococcal disease in the era of intrapartum antibiotic prophylaxis. New Engl J Med 2000;342:15-20.

9 Beardsall K, Thompson MH, Mulla RJ. Neonatal group B streptococcal infection in South Bedfordshire 1993-1998. Arch Dis Child Fetal Neonatal Ed 2000;82:F205-7.

10 Bignardi GE. Surveillance of neonatal group B streptococcal infection in Sunderland. Commun Dis Public Health 1999;2:264-5.

11 Moses LM, Heath PT, Wilkinson AR, Jeffery HE, Isaacs D. Early onset group B streptococcal neonatal infection in Oxford 1985-96. Arch Dis Child Fetal Neonatal Ed 1998;79:F148-49.

12 Fey R, Stuart J, George R. Neonatal group B streptococcal infection in England and Wales 1981-1997. Arch Dis Child Fetal Neonatal Ed 1999:80(suppl 1):A70,G202.

13 d'Agapeyeff K, Torry MJ, Luck S, Pitt A, Bell A, Heath PT, Bedford Russell A. Culture positive early onset group B streptococcal disease- -the tip of the iceberg. Arch Dis Child 2001;84(suppl 1):A30-31.

14 Heath PT, Balfour G, Tighe H, Efstratiou A, Kaye P, Rennie J, et al. Group B streptococcal disease (GBS) in UK infants less than 90 days of age: a national surveillance study. Arch Dis Child 2001;84(suppl 1):A8.

15 Webber S, Wilkinson AR, Lindsell D, Hope PL, Dobson SR, Isaacs D. Neonatal pneumonia. Arch Dis Child 1990;65:207-11.

16 Perinatal mortality: a continuing collaborative regional survey. BMJ 1984;288:1717-20

17 Confidential Enquiry into Stillbirths and Deaths in Infancy. 7th annual report. London: Maternal and Child Health Consortium, 2000 (http://www.cesdi.org.uk/publications/7th_ExecSummary.pdf).

18 Embleton N, Wariyar U, Hey E. Mortality from early onset group B streptococcal infection in the United Kingdom. Arch Dis Child Fetal Neonatal Ed 1999;80:F139-41. 
19 De Cueto M, Sanchez MJ, Sampedro A, Miranda JA, Herruzo AJ, Rosa-Fraile M. Timing of intrapartum ampicillin and prevention of vertical transmission of group B streptococcus. Obstet Gynecol 1998;91:112-4.

20 Pylipow M, Gaddis M, Kinney JS. Selective intrapartum prophylaxis for group B streptococcus colonisation: management and outcome of newborns. Pediatrics 1994;93:631-5.

21 Benitz WE, Gould JB, Druzin ML. Risk factors for early-onset group B streptococcal sepsis: estimation of odds ratios by critical literature review. Pediatrics 1999;103:e77

22 Benitz WE, Gould JB, Druzin ML. Antimicrobial prevention of early-onset group B streptococcal sepsis: estimates of risk reduction based on a critical literature review. Pediatrics 1999;103:e79.
23 Joseph TA, Pyati SP, Jacobs N. Neonatal early onset Escherichia coli disease: the effect of intrapartum ampicillin. Arch Pediatr Adolesc Med 1998;152:35-40.

24 Kenyon S, Taylor D, Tarnow-Mordi W. Broad-spectrum antibiotics for preterm, prelabour rupture of fetal membranes: the ORACLE I randomised trial. Lancet 2001;357:979-88.

(Accepted 12 February 2002)

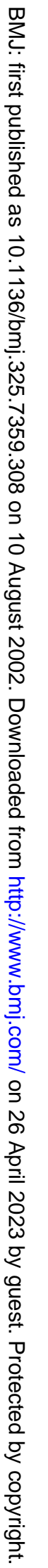

